\title{
Asymmetric masks for large field-of-view and high- energy X-ray phase contrast imaging
}

\author{
M. Endrizzi, ${ }^{\mathrm{a}}$ A. Astolfo, ${ }^{\mathrm{a}}$ B. Price, ${ }^{\mathrm{b}}$ I. Haig, ${ }^{\mathrm{b}}$ and A. Olivo ${ }^{\mathrm{a}}$ \\ ${ }^{a}$ Department of Medical Physics and Biomedical Engineering, University College London, Gower \\ Street, London WC1E 6BT, United Kingdom \\ ${ }^{b}$ Nikon Metrology UK Ltd., Unit 5, Icknield Industrial Estate, Tring, Herts HP23 4JX, United Kingdom \\ E-mail:m.endrizzi@ucl.ac.uk
}

\begin{abstract}
We report on a large field of view, laboratory-based X-ray phase-contrast imaging setup. The method is based upon the asymmetric mask design that enables the retrieval of the absorption, refraction and scattering properties of the sample without the need to move any component of the imaging system. This can be thought of as a periodic repetition of a group of three (or more) apertures arranged in such a way that each laminar beam, defined by the apertures, produces a different illumination level when analysed with a standard periodic set of apertures. The sample is scanned through the imaging system, also removing possible aliasing problems that might arise from partial sample illumination when using the edge illumination technique. This approach preserves the incoherence and achromatic properties of edge illumination, removes the problems related to aliasing and it naturally adapts to those situations in clinical, industrial and security imaging where the image is acquired by scanning the sample relative to the imaging system. These concepts were implemented for a large field-of-view set of masks $(20 \mathrm{~cm} \times 1.5 \mathrm{~cm}$ and $15 \mathrm{~cm} \times 1.2 \mathrm{~cm})$, designed to work with a tungsten anode X-ray source operated up to $80-100 \mathrm{kVp}$, from which preliminary experimental results are presented.
\end{abstract}

KEYWORDS: X-ray imaging; phase-contrast imaging; asymmetric masks. 


\section{Contents}

1. Introduction 1

2. Methods 1

3. Results $\quad 3$

$\begin{array}{lr}\text { 4. Conclusion } & 5\end{array}$

\section{Introduction}

$\mathrm{X}$-ray imaging is extremely widely used as a non-destructive method for visualizing the inner structure of extended objects. It finds application across a range of field, from biology and medicine to security and engineering. The capability of generating image contrast based on the difference in the attenuation of the X-rays as they travel through the sample can be extended by means of phase-contrast techniques were also the modulations imparted to the phase of the wavefield are contributing to the intensity contrast at the detector [1-3]. A variety of techniques are available today to perform X-ray phase-contrast imaging (XPCI). These include, amongst others, crystal interferometry and analysers [4-13], propagation in free space [14, 15], grating interferometry [16-18] and edge illumination [19, 20]. Edge illumination (EI) is attractive for its compatibility with the polychromatic and divergent X-ray beam produced by conventional tubes. It can provide quantitative phase and scattering information, and offers a robust set-up [21-28].

\section{Methods}

Two sets of apertures are at the core of the EI XPCI technique. The first one is often called presample mask and is used for beam shaping, while the second one, usually called detector mask, is used for beam analysis and the sample is positioned between the two masks. The intensity that reaches the detector depends on the relative alignment of these two optical elements: if the apertures are perfectly aligned the intensity is at its maximum and it progressively decreases as one mask is shifted with respect to the other. We refer to the function describing the intensity as a function of the relative displacement between the apertures in the two masks the system's illumination function. The conventional and the asymmetric [27] mask designs for EI XPCI are sketched in Figure 1. In the conventional design a regular pattern, alternating between transmitting and absorbing septa, is used in both the pre-sample and in the detector masks. This enables to obtain a uniform illumination level across the entire field of view and it can be schematized with the system working at a given position along its illumination function. In the general case, at least three images (intensity projections) - at complementary illumination fractions - are required in order to retrieve the sample's absorption, refraction and scattering images [22,27]. 

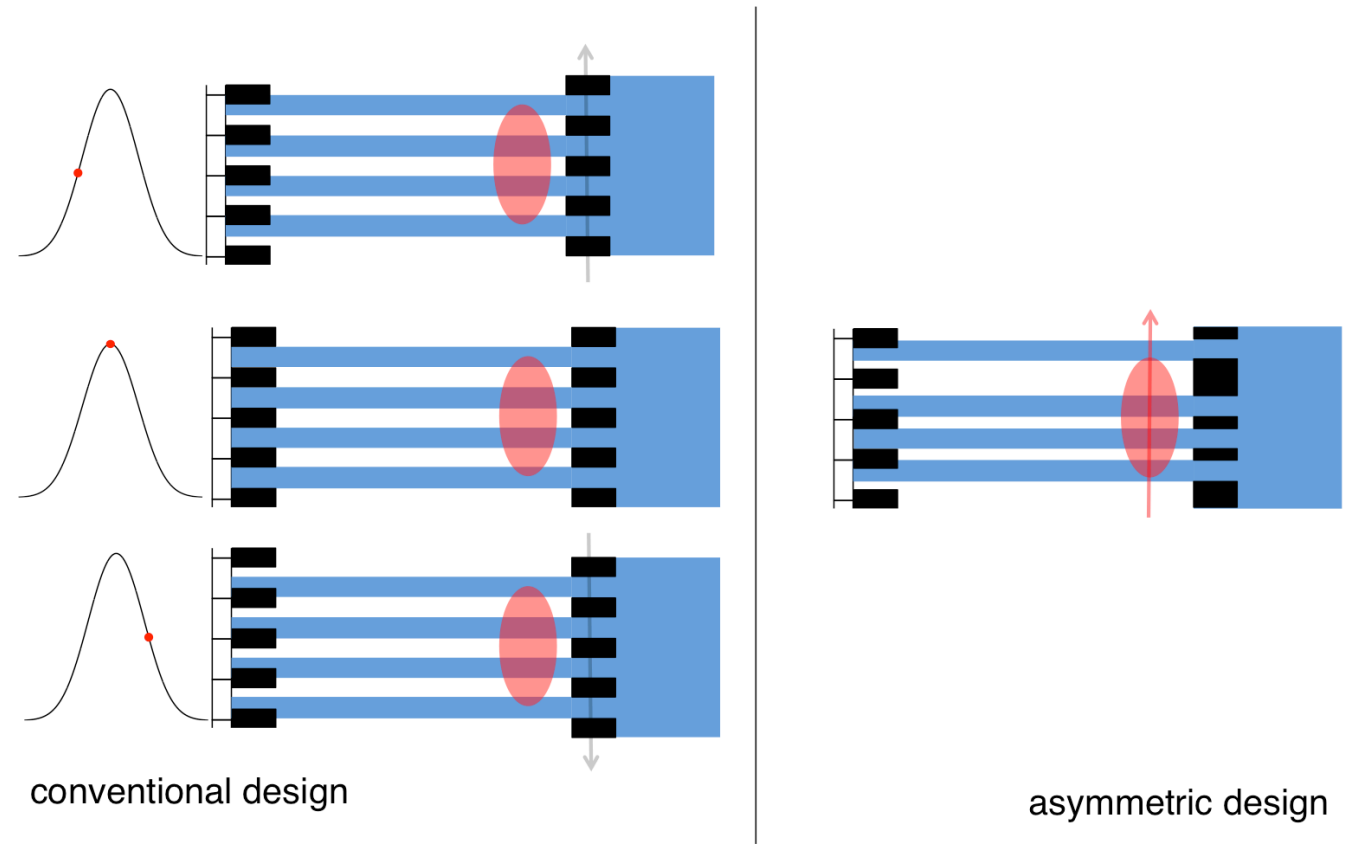

asymmetric design

Fig. 1: Comparison between the mask designs. Conventional: three separate images are acquired at different illumination levels by moving one mask with respect to the other. Asymmetric: the intensity projections are acquired by adjacent pixel columns, each reached by complementary illumination fractions. In the asymmetric configuration, the sample is scanned and the optical elements are kept stationary.

Three typical positions are at about $50 \%$ of the intensity on each side of the illumination function and at $100 \%$. We note that the masks have to be moved during data collection in order to perform this kind of imaging, and this could be impractical in some situations, for example were system robustness has to be maximised. An alternative to this configuration is to use an asymmetric pattern of apertures and absorbing septa in the sample mask while keeping a regular pattern in the detector mask. In EI there is a one-to-one matching between each aperture in the two masks and the detector pixel columns. When an asymmetric mask is used, each pixel column records independently an intensity projection at complementary illumination fractions. The sample is scanned through the X-ray beam while all the other components of the system are kept stationary during data acquisition. With this concept it is possible to extend the field of view of an XPCI system by scaling the masks only in one direction, the extension of the image in the direction orthogonal to the apertures does not depend on the masks' size any more, but is only limited by the amount of scanning that is feasible to perform. Based on this concept a system featuring a sample mask of $15 \mathrm{~cm} \times 1.2 \mathrm{~cm}$ was designed and assembled [29]. The large mask was obtained by combining three smaller masks $(5 \mathrm{~cm} \times 1.2 \mathrm{~cm}$ each) that were accurately aligned and fixed to a steel frame. The apertures were chosen to be 22 and 28 microns for the sample and the detector mask, respectively. The X-ray source is a tungsten anode X-tek 160 tube with a focal spot of approximately 80 microns. The detector is a dual-energy, singlephoton-counting CdTe-CMOS (XCounter) featuring 2048 x 128 pixel of 100 microns size. The entire system is assembled inside a lead shielded X-tek NGI (Next Generation Imaging) cabinet. Preliminary results obtained from this scanner are presented in the following section. 


\section{Results}

A photograph of the scanning system is shown in Figure 2 where the sample mask, the sample stage, the detector mask and the detector are visible. Alignment of the two masks with the detector pixel columns is achieved by means of two stacks of stepper motors.

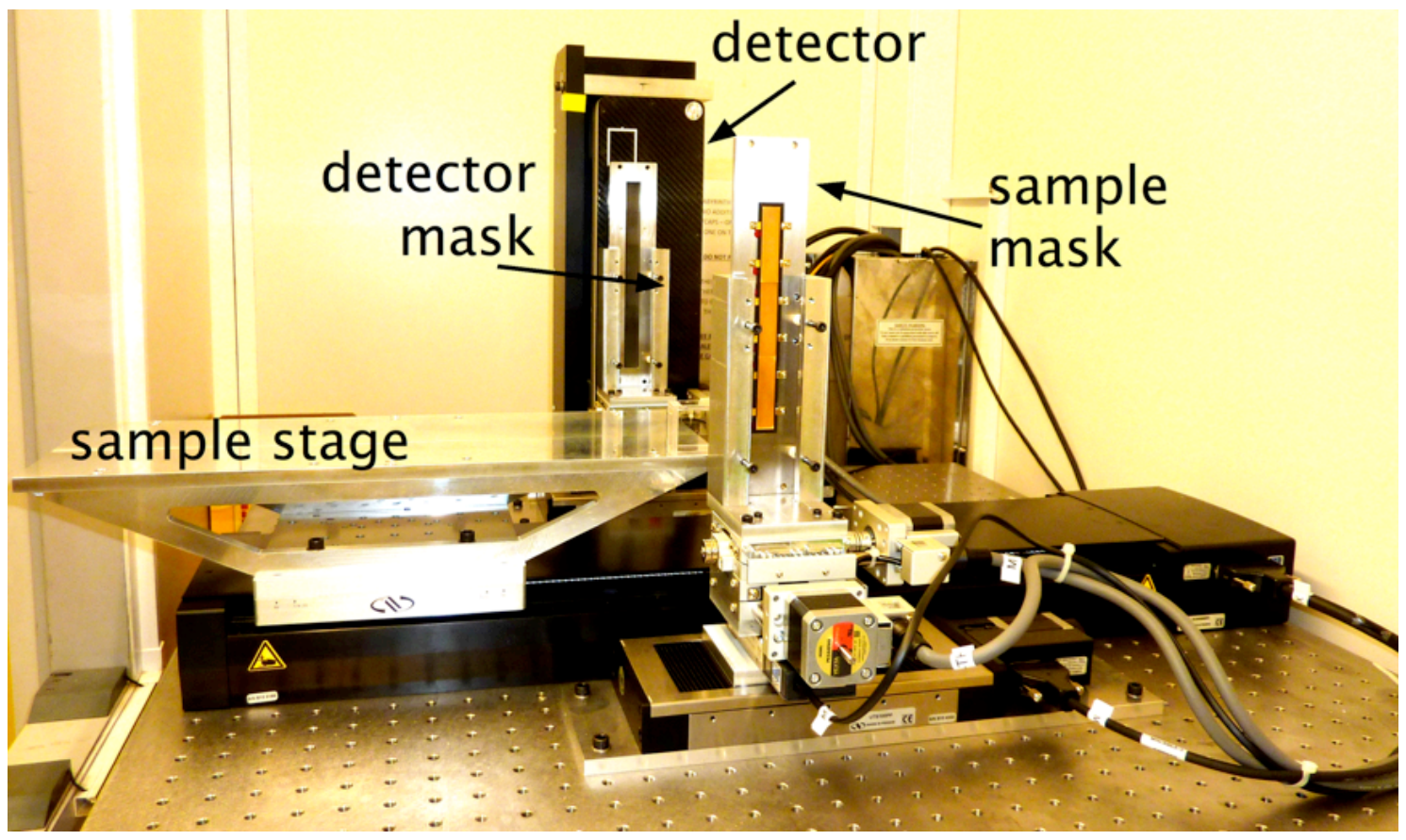

Fig. 2: Photograph of the scanning system prototype. The sample mask is $15 \mathrm{~cm} \times 1.2 \mathrm{~cm}$ in size and the sample stage can scan large samples. The single-photon-counting detector is visible behind the detector mask.

The asymmetric mask is designed to provide four complementary illumination conditions, approximately at 100\%, 60\% (two sides) and 20\% (one side). The curves recorded experimentally are shown in Figure 3. 


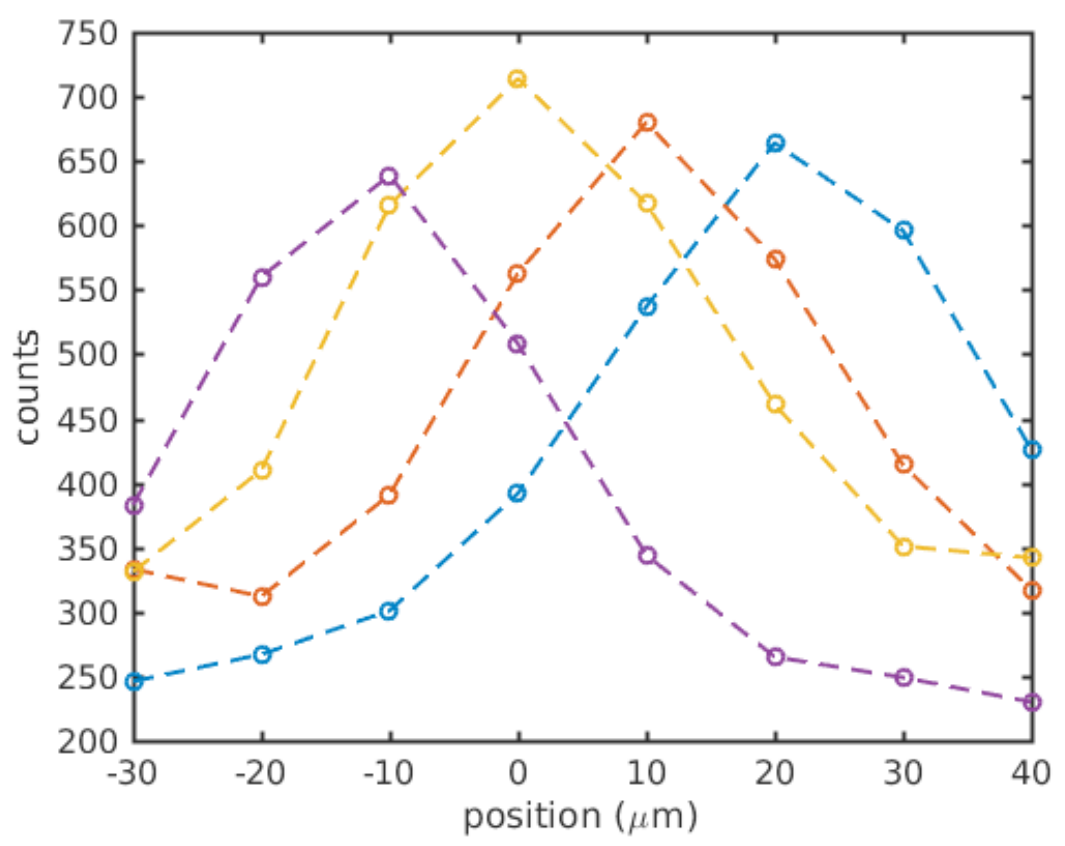

Fig. 3: Illumination function simultaneously recorded by adjacent pixel columns: at the position with no relative displacement, the illumination fractions are approximately $100 \%, 60 \%$ (two sides) and $20 \%$ (one side).

Finally we report on the retrieval of three contrast channels: absorption, refraction and scattering of a marker pen used as test sample. The marker was placed vertically inside the scanner such that the shorter dimension is orthogonal to the masks' aperture and parallel to the scanning direction. The retrieval is based on a multi-Gaussian model of the illumination function and on the extraction of the sample parameters on a pixel-by-pixel basis by means of a fitting procedure [29]. The illumination function is modelled as

$L(x)=\sum_{i=1}^{4} a_{i 0} \frac{1}{\sqrt{2 \pi a_{i 2}^{2}}} \exp \left[-\frac{1}{2} \frac{\left(x-a_{i 1}\right)^{2}}{a_{i 2}^{2}}\right]+a_{3}$

and the sample transmission $t$, refraction $\Delta$ and scattering $\sigma$ are extracted by fitting the data to the modified illumination function

$G(x)=\sum_{i=1}^{4} a_{i 0} \frac{t_{1}}{\sqrt{2 \pi\left(a_{i 2}^{2}+\sigma^{2}\right)}} \exp \left[-\frac{1}{2} \frac{\left(x-a_{i 1}-\Delta\right)^{2}}{a_{i 2}^{2}+\sigma^{2}}\right]+t_{0} a_{3}$

that describes how the illumination is modified when the sample is placed in the X-ray beam. The three images are reported in Figure 4: a) transmission, b) refraction and c) scattering, where the complementarity of these three contrast mechanisms can be observed. Some line-type artefact and imperfections can be seen in the images, which are due to defects in the masks' 
structure. In these location the illumination model is not accurate anymore and this prevents the correct retrieval of the sample parameters.

a)

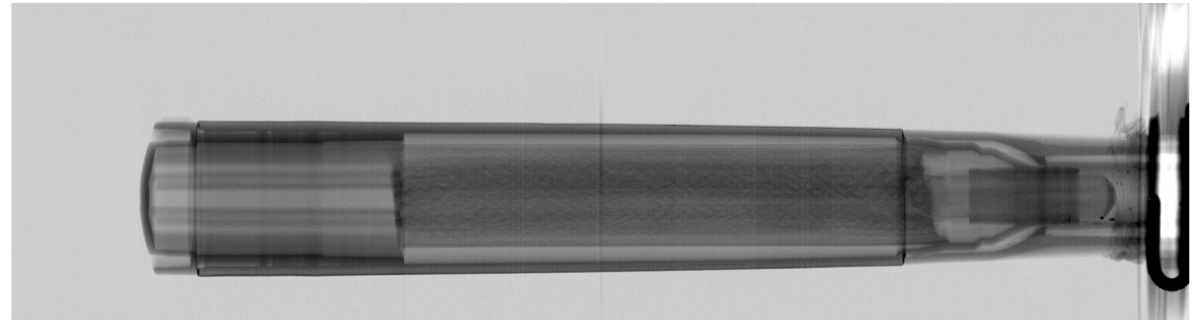

b)

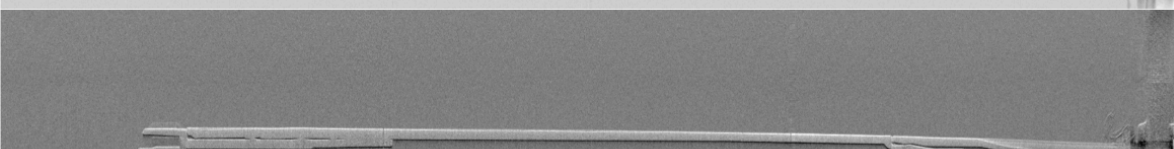

c)

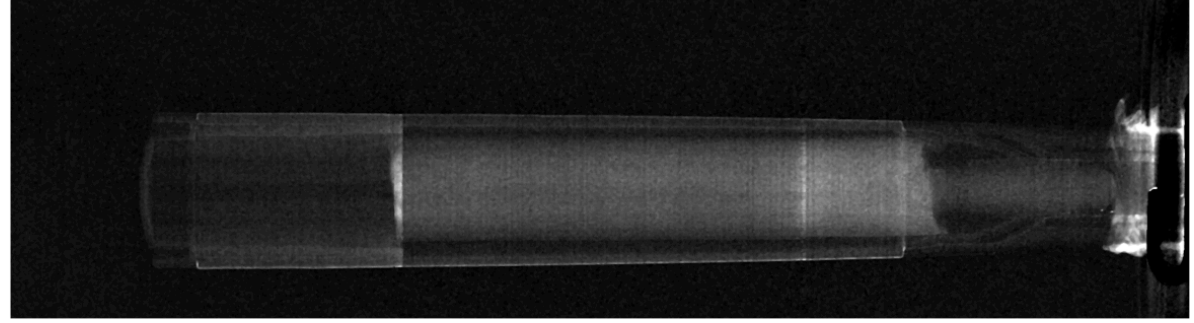

Fig. 4: a) transmission, b) refraction and c) scattering images of a marker pen test object. The sample was placed with the longer dimension parallel to the masks' apertures such that the sample was scanned along the shortest dimension.

\section{Conclusion}

We presented the concept of asymmetric masks for edge illumination X-ray phase-contrast imaging and we showed how the resulting imaging system can provide absorption, refraction and scattering contrast imaging by requiring the only movement of the sample. This can be advantageous in those situations were the accurate movement of optical elements during data acquisition is not feasible or impractical, for example due to robustness constraints on the system. A large field-of-view prototype scanning system, based on this new mask concept, was recently built. Preliminary results obtained on the new scanner were presented.

\section{Acknowledgments}

This project was funded under the Innovative Research Call in Explosives and Weapons Detection 2013 initiative. This is a Cross-Government programme sponsored by a number of Departments and Agencies under the UK Governments CONTEST strategy in partnership with the US Department of Homeland Security, Science and Technology Directorate. Additional support to this work was provided by the UK Engineering and Physical Sciences Research 
Council Grant EP/I021884/1. ME was supported by the Royal Academy of Engineering under the RAEng Research Fellowships scheme.

\section{References}

[1] R. Fitzgerald, "Phase-sensitive x-ray imaging," Physics Today 53(7), pp. 23-26, 2000.

[2] A. Bravin, P. Coan, and P. Suortti, "X-ray phase-contrast imaging: from pre-clinical applications towards clinics," Physics in Medicine and Biology 58(1), p. R1, 2013.

[3] S. Wilkins, Y. I. Nesterets, T. Gureyev, S. Mayo, A. Pogany, and A. Stevenson, "On the evolution and relative merits of hard x-ray phase-contrast imaging methods," Philosophical Transactions of the Royal Society of London A: Mathematical, Physical and Engineering Sciences 372(2010), p. $20130021,2014$.

[4] U. Bonse and M. Hart, “An x-ray interferometer,” Applied Physics Letters 6, pp. 155 -156, 1965.

[5] T. J. Davis, D. Gao, T. E. Gureyev, A. W. Stevenson, and S. W. Wilkins, "Phase-contrast imaging of weakly absorbing materials using hard X-rays,” Nature 373, pp. 595-598, 1995.

[6] A. Momose, T. Takeda, Y. Itai, and K. Hirano, "Phase-contrast x-ray computed tomography for observing biological soft tissues,” Nature Medicine 2(4), pp. 473-475, 1996.

[7] K. Goetz, E. Foerster, P. Zaumseil, M. P. Kalashnikov, I. A. Mikhailov, G. V. Sklizkov, and S. I. Fedotov, "Measurements of the parameters of shell targets for laser thermonuclear fusion using an Xray schlieren method,” Kvantovaia Elektronika Moscow 6, pp. 1037-1042, 1979.

[8] E. Foerster, K. Goetz, and P. Zaumseil, "Double crystal diffractometry for the characterization of targets for laser fusion experiments," Kristall und Technik 15(8), pp. 937-945, 1980.

[9] V. N. Ingal and E. A. Beliaevskaya, "X-ray plane-wave topography observation of the phase contrast from a non-crystalline object," Journal of Physics D Applied Physics 28, pp. 2314-2317, 1995.

[10] D. Chapman, W. Thomlinson, R. E. Johnston, D. Washburn, E. Pisano, N. Gmü, Z. Zhong, R. Menk, F. Arfelli, and D. Sayers, "Diffraction enhanced x-ray imaging," Physics in Medicine and Biology 42, pp. 2015-2025, 1997.

[11] L. Rigon, H.-J. Besch, F. Arfelli, R.-H. Menk, G. Heitner, and H. Plothow-Besch, “A new DEI algorithm capable of investigating sub-pixel structures," Journal of Physics D Applied Physics 36(26), pp. A260000-A112, 2003.

[12] E. Pagot, P. Cloetens, S. Fiedler, A. Bravin, P. Coan, J. Baruchel, J. H“artwig, and W. Thomlinson, "A method to extract quantitative information in analyzer-based x-ray phase contrast imaging," Applied Physics Letters 82, p. 3421, 2003.

[13] M. N. Wernick, O. Wirjadi, D. Chapman, Z. Zhong, N. P. Galatsanos, Y. Yang, J. G. Brankov, O. Oltulu, M. A. Anastasio, and C. Muehleman, "Multiple-image radiography," Physics in Medicine and Biology 48, pp. 3875-3895, 2003.

[14] A. Snigirev, I. Snigireva, V. Kohn, S. Kuznetsov, and I. Schelokov, "On the possibilities of x-ray phase contrast microimaging by coherent high-energy synchrotron radiation," Review of Scientific Instruments 66(12), pp. 5486-5492, 1995. 
[15] S. W. Wilkins, T. E. Gureyev, D. Gao, A. Pogany, and A. W. Stevenson, "Phase-contrast imaging using polychromatic hard x-rays," Nature 384, pp. 335-338, 1996.

[16] C. David, B. Nohammer, H. H. Solak, and E. Ziegler, "Differential x-ray phase contrast imaging using a shearing interferometer," Applied Physics Letters 81(17), pp. 3287-3289, 2002.

[17] T. Weitkamp, A. Diaz, C. David, F. Pfeiffer, M. Stampanoni, P. Cloetens, and E. Ziegler, "X-ray phase imaging with a grating interferometer," Optics Express 13, pp. 6296-6304, Aug 2005.

[18] F. Pfeiffer, T. Weitkamp, O. Bunk, and C. David, "Phase retrieval and differential phase-contrast imaging with low-brilliance X-ray sources," Nature Physics 2, pp. 258-261, 2006.

[19] A. Olivo, F. Arfelli, G. Cantatore, R. Longo, R. H. Menk, S. Pani, M. Prest, P. Poropat, L. Rigon, G. Tromba, E. Vallazza, and E. Castelli, "An innovative digital imaging set-up allowing a low-dose approach to phase contrast applications in the medical field," Medical Physics 28, pp. 1610-1619, 2001.

[20] A. Olivo and R. Speller, "A coded-aperture technique allowing x-ray phase contrast imaging with conventional sources,” Applied Physics Letters 91(7), p. 074106, 2007.

[21] P. R. Munro, K. Ignatyev, R. D. Speller, and A. Olivo, "Phase and absorption retrieval using incoherent X-ray sources,” Proc. Natl. Acad. Sci. USA 109(35), pp. 13922-13927, 2012.

[22] M. Endrizzi, P. C. Diemoz, T. P. Millard, J. L. Jones, R. D. Speller, I. K. Robinson, and A. Olivo, "Hard x-ray dark-field imaging with incoherent sample illumination," Applied Physics Letters 104(2), p. 024106, 2014.

[23] M. Endrizzi, F. A. Vittoria, G. Kallon, D. Basta, P. C. Diemoz, A. Vincenzi, P. Delogu, R. Bellazzini, and A. Olivo, "Achromatic approach to phase-based multi-modal imaging with conventional x-ray sources,” Optics Express 23(12), pp. 16473-16480, 2015.

[24] T. P. Millard, M. Endrizzi, K. Ignatyev, C. K. Hagen, P. R. T. Munro, R. D. Speller, and A. Olivo, "Method for automatization of the alignment of a laboratory based x-ray phase contrast edge illumination system," Review of Scientific Instruments 84(8), p. 083702, 2013.

[25] M. Endrizzi, D. Basta, and A. Olivo, "Laboratory-based x-ray phase-contrast imaging with misaligned optical elements," Applied Physics Letters 107(12), p. 124103, 2015.

[26] M. Endrizzi and A. Olivo, "Absorption, refraction and scattering retrieval with an edgeillumination-based imaging setup," Journal of Physics D: Applied Physics 47(50), p. 505102, 2014.

[27] M. Endrizzi, A. Astolfo, F. A. Vittoria, T. P. Millard, and A. Olivo, "Asymmetric masks for laboratory-based x-ray phase-contrast imaging with edge illumination,” Scientific reports 6, 2016.

[28] MC Longo, L. Rigon, F.C.M. Lopez, R. Chen, D. Dreossi, F. Zanconati and R. Longo. "A simplified edge illumination set-up for quantitative phase contrast mammography with synchrotron radiation at clinical doses" Physics in medicine and biology 60.3: N21, 2015

[29] A. Astolfo, M. Endrizzi, B. Price, I. Haig and A. Olivo, "The first large area, high x-ray energy phase contrast prototype for enhanced detection of threat object in baggage screening" SPIE Proceedings in press 